\title{
Global Behavior of Two Rational Third Order Difference Equations
}

\author{
R. Abo-Zeid ${ }^{1 *}$ and H. Kamal ${ }^{1}$ \\ ${ }^{1}$ Department of Basic Science, The Higher Institute for Engineering \& Technology, Al-Obour, Cairo, Egypt \\ *Corresponding author
}

\section{Article Info}

Keywords: Difference equation, Forbidden set, Periodic solution.

2010 AMS: $39 A 20$

Received: 29 September 2019

Accepted: 5 November 2019

Available online: 26 December 2019

\section{Abstract}

In this paper, we solve and study the global behavior of all admissible solutions of the two difference equations

and

$$
x_{n+1}=\frac{x_{n} x_{n-2}}{x_{n-1}-x_{n-2}}, \quad n=0,1, \ldots
$$

$$
x_{n+1}=\frac{x_{n} x_{n-2}}{-x_{n-1}+x_{n-2}}, \quad n=0,1, \ldots,
$$

where the initial values $x_{-2}, x_{-1}, x_{0}$ are real numbers.

We show that every admissible solution for the first equation converges to zero. For the other equation, we show that every admissible solution is periodic with prime period six. Finally we give some illustrative examples.

\section{Introduction}

In [11], the author determined the forbidden sets and discussed the global behaviors of solutions of the two difference equations

$$
x_{n+1}=\frac{x_{n} x_{n-1}}{x_{n}-x_{n-2}}, \quad n=0,1, \ldots,
$$

and

$$
x_{n+1}=\frac{x_{n} x_{n-1}}{-x_{n}+x_{n-2}}, \quad n=0,1, \ldots,
$$

where the initial values $x_{-2}, x_{-1}, x_{0}$ are real numbers.

In [2], the author determined the forbidden sets and discussed the global behaviors of solutions of the two difference equations

$$
x_{n+1}=\frac{a x_{n} x_{n-1}}{ \pm b x_{n-1}+c x_{n-2}}, \quad n=0,1, \ldots,
$$

where $a, b, c$ are positive real numbers and the initial conditions $x_{-2}, x_{-1}, x_{0}$ are real numbers.

Elsayed in [19] studied the behavior of solutions of the nonlinear difference equation

$$
x_{n+1}=a x_{n-1}+\frac{b x_{n} x_{n-1}}{c x_{n}+d x_{n-2}}, \quad n=0,1, \ldots,
$$

where $a, b, c, d$ are positive real constants and the initial conditions $x_{-2}, x_{-1}, x_{0}$ are arbitrary positive real numbers. For more on difference equations (See [1,3-10,12-18,20-28]) and the references therein.

In this paper, we study the two difference equations

$$
x_{n+1}=\frac{x_{n} x_{n-2}}{x_{n-1}-x_{n-2}}, \quad n=0,1, \ldots
$$


and

$$
x_{n+1}=\frac{x_{n} x_{n-2}}{-x_{n-1}+x_{n-2}}, \quad n=0,1, \ldots,
$$

where the initial values $x_{-2}, x_{-1}, x_{0}$ are real numbers.

2. The difference equation $x_{n+1}=\frac{x_{n} x_{n-2}}{x_{n-1}-x_{n-2}}$

During this section, we suppose that

$$
\lambda_{-}=\frac{1}{2}-\frac{\sqrt{5}}{2} \text { and } \lambda_{+}=\frac{1}{2}+\frac{\sqrt{5}}{2} \text {. }
$$

\subsection{Solution of Equation (1.1)}

The transformation

$$
y_{n}=\frac{x_{n-1}}{x_{n}}, \text { with } y_{-1}=\frac{x_{-2}}{x_{-1}}, y_{0}=\frac{x_{-1}}{x_{0}}
$$

reduces Equation (1.1) into the difference equation

$$
y_{n+1}=\frac{1}{y_{n-1}}-1, n=0,1, \ldots
$$

By solving Equation (2.2) and after some calculations, the solution of Equation (1.1) can be obtained.

Theorem 2.1. Let $\left\{x_{n}\right\}_{n=-2}^{\infty}$ be an admissible solution of Equation (1.1). Then

$$
x_{n}=\left\{\begin{aligned}
-\frac{v}{\left(x_{0} f_{\frac{n-1}{2}}-x_{-1} f_{\frac{n+1}{2}}\right)\left(x_{-1} f_{\frac{n+1}{2}}-x_{-2} f_{\frac{n+3}{2}}\right)}, & n=1,3, \ldots, \\
\frac{v}{\left(x_{0} f_{\frac{n}{2}}-x_{-1} f_{\frac{n}{2}+1}\right)\left(x_{-1} f_{\frac{n}{2}}-x_{-2} f_{\frac{n}{2}+1}\right)}, & n=2,4, \ldots,
\end{aligned}\right.
$$

where $v=x_{0} x_{-1} x_{-2}$ and $f_{n}$ is the solution of the difference equation

$$
f_{n+2}=f_{n}+f_{n+1}, f_{0}=0, f_{1}=1, n=0,1, \ldots
$$

Proof. We can write the solution formula (2.3) as

$$
x_{2 m+1}=-\frac{v}{\left(x_{0} f_{m}-x_{-1} f_{m+1}\right)\left(x_{-1} f_{m+1}-x_{-2} f_{m+2}\right)}
$$

and

$$
x_{2 m+2}=\frac{v}{\left(x_{0} f_{m+1}-x_{-1} f_{m+2}\right)\left(x_{-1} f_{m+1}-x_{-2} f_{m+2}\right)} .
$$

When $m=0$,

$$
\begin{aligned}
x_{1} & =-\frac{v}{\left(x_{0} f_{0}-x_{-1} f_{1}\right)\left(x_{-1} f_{1}-x_{-2} f_{2}\right)} \\
& =\frac{v}{x_{-1}\left(x_{-1}-x_{-2}\right)}=\frac{x_{0} x_{-2}}{x_{-1}-x_{-2}} .
\end{aligned}
$$

Similarly

$$
\begin{aligned}
x_{2} & =\frac{v}{\left(x_{0} f_{1}-x_{-1} f_{2}\right)\left(x_{-1} f_{1}-x_{-2} f_{2}\right)} \\
& =\frac{v}{\left(x_{0}-x_{-1}\right)\left(x_{-1}-x_{-2}\right)}=\frac{x_{1} x_{-1}}{x_{0}-x_{-1}} .
\end{aligned}
$$

Suppose that the solution formula (2.4) is true for $m>0$. Then

$$
\begin{aligned}
\frac{x_{2 m+1} x_{2 m-1}}{x_{2 m}-x_{2 m-1}} & =\frac{\left(\frac{v}{\left(x_{0} f_{m}-x_{-1} f_{m+1}\right)\left(x_{-1} f_{m+1}-x_{-2} f_{m+2}\right)}\right)\left(\frac{v}{\left(x_{0} f_{m-1}-x_{-1} f_{m}\right)\left(x_{-1} f_{m}-x_{-2} f_{m+1}\right)}\right)}{\frac{v}{\left(x_{0} f_{m}-x_{-1} f_{m+1}\right)\left(x_{-1} f_{m}-x_{-2} f_{m+1}\right)}+\frac{v}{\left(x_{0} f_{m-1}-x_{-1} f_{m}\right)\left(x_{-1} f_{m}-x_{-2} f_{m+1}\right)}} \\
& =\frac{v}{\left(x_{0} f_{m-1}-x_{-1} f_{m}\right)\left(x_{-1} f_{m+1}-x_{-2} f_{m+2}\right)+\left(x_{0} f_{m}-x_{-1} f_{m+1}\right)\left(x_{-1} f_{m+1}-x_{-2} f_{m+2}\right)} \\
& =\frac{v}{\left(x_{-1} f_{m+1}-x_{-2} f_{m+2}\right)\left(x_{0}\left(f_{m-1}+f_{m}\right)-x_{-1}\left(f_{m}+f_{m+1}\right)\right)} \\
& =\frac{v}{\left(x_{-1} f_{m+1}-x_{-2} f_{m+2}\right)\left(x_{0} f_{m+1}-x_{-1} f_{m+2}\right)} \\
& =x_{2 m+2} .
\end{aligned}
$$

Similarly we can show that

$$
\frac{x_{2 m+2} x_{2 m}}{a x_{2 m+1}+b x_{2 m}}=x_{2 m+3} \text {. }
$$

This completes the proof. 
It is clear for Equation (1.1) that if we start with the point $\left(x_{0}, x_{-1}, x_{-2}\right) \in \mathbb{R}^{3}$, we have the following:

If $x_{0}=0$ and $x_{-1} x_{-2} \neq 0$, then $x_{3}$ is undefined.

If $x_{-1}=0$ and $x_{0} x_{-2} \neq 0$, then $x_{5}$ is undefined.

If $x_{-2}=0$ and $x_{0} x_{-1} \neq 0$, then $x_{4}$ is undefined.

Therefore, any point $\left(x_{0}, x_{-1}, x_{-2}\right) \in \mathbb{R}^{3}$ with $x_{0} x_{-1} x_{-2}=0$ belongs to the forbidden set of Equation (1.1).

The following result provides the forbidden set of Equation (1.1).

Theorem 2.2. The forbidden set of equation (1.1) is

$$
\begin{aligned}
F= & \bigcup_{i=0}^{2}\left\{\left(u_{0}, u_{-1}, u_{-2}\right) \in \mathbb{R}^{3}: u_{-i}=0\right\} \cup \bigcup_{m=1}^{\infty}\left\{\left(u_{0}, u_{-1}, u_{-2}\right) \in \mathbb{R}^{3}: u_{0}=u_{-1} \frac{f_{m+1}}{f_{m}}\right\} \cup \\
& \bigcup_{m=1}^{\infty}\left\{\left(u_{0}, u_{-1}, u_{-2}\right) \in \mathbb{R}^{3}: u_{-1}=u_{-2} \frac{f_{m+1}}{f_{m}}\right\} .
\end{aligned}
$$

Proof. The proof is clear using the arguments after Theorem (2.1) and formula (2.3).

\subsection{Global behavior of equation (1.1)}

In this section, we shall give two invariant sets for Equation (1.1) and a result concerns the global behavior of the solutions of Equation (1.1). Consider the set

$$
D_{1}=\left\{(x, y, z) \in \mathbb{R}^{3}: \frac{x}{1 / \lambda_{-}^{2}}=-\frac{y}{1 / \lambda_{-}}=z\right\}
$$

and

$$
D_{2}=\left\{(x, y, z) \in \mathbb{R}^{3}: \frac{x}{1 / \lambda_{+}^{2}}=-\frac{y}{1 / \lambda_{+}}=z\right\}
$$

Theorem 2.3. The two sets $D_{1}$ and $D_{2}$ are invariant sets for Equation (1.1).

Proof. Let $\left(x_{0}, x_{-1}, x_{-2}\right) \in D_{1}$. We show that $\left(x_{n}, x_{n-1}, x_{n-2}\right) \in D_{1}$ for each $n \in \mathbb{N}$. The proof is by induction on $n$. The point $\left(x_{0}, x_{-1}, x_{-2}\right) \in$ $D_{1}$ implies

$$
\frac{x_{0}}{1 / \lambda_{-}^{2}}=-\frac{x_{-1}}{1 / \lambda_{-}}=x_{-2}
$$

Now for $n=1$, we have

$$
x_{1}=\frac{x_{0} x_{-2}}{x_{-1}-x_{-2}}=\frac{\left(1 / \lambda_{-}\right) x_{-1} \lambda_{-} x_{-1}}{x_{-1}+\lambda_{-} x_{-1}}=\frac{x_{-1}}{\lambda_{-}^{2}} .
$$

Then we have

$$
\frac{x_{1}}{1 / \lambda_{-}^{2}}=-\frac{x_{0}}{1 / \lambda_{-}}=x_{-1}
$$

This implies that $\left(x_{1}, x_{0}, x_{-1}\right) \in D_{1}$.

Suppose now that $\left(x_{n}, x_{n-1}, x_{n-2}\right) \in D_{1}$. This means that

$$
\frac{x_{n}}{1 / \lambda_{-}^{2}}=-\frac{x_{n-1}}{1 / \lambda_{-}}=x_{n-2} \text {. }
$$

Then

$$
x_{n+1}=\frac{x_{n} x_{n-2}}{x_{n-1}-x_{n-2}}=\frac{\left(1 / \lambda_{-}\right) x_{n-1} \lambda_{-} x_{n-1}}{x_{n-1}+\lambda_{-} x_{n-1}}=\frac{x_{n-1}}{\lambda_{-}^{2}} .
$$

This implies that $\left(x_{n+1}, x_{n}, x_{n-1}\right) \in D_{1}$. Therefore, $D_{1}$ is an invariant set for Equation (1.1).

By similar way, we can show that $D_{2}$ is an invariant set for Equation (1.1).

This completes the proof.

Theorem 2.4. Every admissible solution of Equation (1.1) converges to zero.

Proof. Suppose that $\left\{x_{n}\right\}_{n=-2}^{\infty}$ is an admissible solution of Equation (1.1).

Using Formula (2.4), we can write

$$
\begin{aligned}
x_{2 m+1} & =-\frac{v}{\left(x_{0} f_{m}-x_{-1} f_{m+1}\right)\left(x_{-1} f_{m+1}-x_{-2} f_{m+2}\right)} \\
& =-\frac{v}{f_{m} f_{m+1}\left(x_{0}-x_{-1} \frac{f_{m+1}}{f_{m}}\right)\left(x_{-1}-x_{-2} \frac{f_{m+2}}{f_{m+1}}\right)} .
\end{aligned}
$$

But

$$
\frac{f_{m+1}}{f_{m}} \rightarrow \lambda_{+} \text {and } f_{m} \rightarrow \infty \text { as } m \rightarrow \infty .
$$


This implies that

$$
x_{2 m+1} \rightarrow 0 \text { as } m \rightarrow \infty .
$$

Similarly, we can show that $x_{2 m+2} \rightarrow 0$, as $m \rightarrow \infty$.

Therefore, $x_{n} \rightarrow 0$ as $n \rightarrow \infty$. This completes the proof.

\section{Example (1)}

Figure (2.1) shows that a solution $\left\{x_{n}\right\}_{n=-2}^{\infty}$ of equation (1.1) with $x_{-2}=2, x_{-1}=-0.2$ and $x_{0}=1$ converges to zero.

\section{Example (2)}

Figure (2.2) shows that a solution $\left\{x_{n}\right\}_{n=-2}^{\infty}$ of equation (1.1) with $x_{-2}=-1, x_{-1}=-0.2$ and $x_{0}=-1.8$ converges to zero.

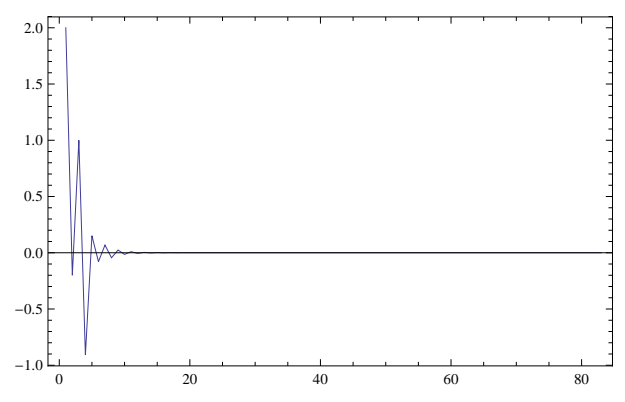

Figure 2.1: $x_{n+1}=\frac{x_{n} x_{n-2}}{x_{n-1}-x_{n-2}}$

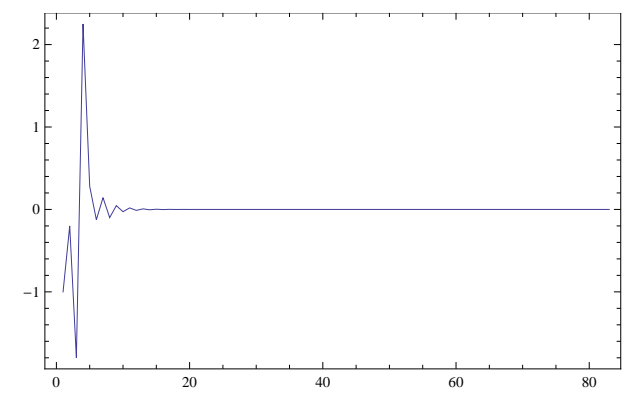

Figure 2.2: $x_{n+1}=\frac{x_{n} x_{n-2}}{x_{n-1}-x_{n-2}}$

\section{The difference equation $x_{n+1}=\frac{x_{n} x_{n-2}}{-x_{n-1}+x_{n-2}}$}

In this section, we study the difference equation (1.2).

\subsection{Solution of Equation (1.2)}

The transformation (2.1) reduces Equation (1.2) into the difference equation

$$
y_{n+1}=-\frac{1}{y_{n-1}}+1, n=0,1, \ldots
$$

By solving Equation (3.1) and after some calculations, the solution of Equation (1.2) can be obtained.

Theorem 3.1. Let $\left\{x_{n}\right\}_{n=-2}^{\infty}$ be an admissible solution of Equation (1.2). Then

$$
x_{n}=\left\{\begin{array}{cl}
\frac{\mu}{\left(\alpha_{0} \cos \frac{(n-3) \pi}{6}-\beta_{0} \sin \frac{(n-3) \pi}{6}\right)\left(\alpha_{-1} \cos \frac{(n-1) \pi}{6}-\beta_{-1} \sin \frac{(n-1) \pi}{6}\right)}, & n=1,3, \ldots, \\
\frac{\left(\alpha_{0} \cos \frac{(n-2) \pi}{6}-\beta_{0} \sin \frac{(n-2) \pi}{6}\right)\left(\alpha_{-1} \cos \frac{(n-2) \pi}{6}-\beta_{-1} \sin \frac{(n-2) \pi}{6}\right)}{6}, & n=2,4, \ldots,
\end{array}\right.
$$

where $\mu=x_{0} x_{-1} x_{-2}, \alpha_{0}=-x_{0}+x_{-1}, \beta_{0}=\frac{1}{\sqrt{3}}\left(x_{0}+x_{-1}\right), \alpha_{-1}=-x_{-1}+x_{-2}$ and $\beta_{-1}=\frac{1}{\sqrt{3}}\left(x_{-1}+x_{-2}\right)$.

Proof. We can write the given solution (3.2) as

$$
x_{2 m+1}=\frac{\mu}{\gamma_{0}(m-1) \gamma_{-1}(m)}
$$

and

$$
x_{2 m+2}=\frac{\mu}{\gamma_{0}(m) \gamma_{-1}(m)},
$$

where

$$
\gamma_{0}(m)=\alpha_{0} \cos \frac{m \pi}{3}-\beta_{0} \sin \frac{m \pi}{3}
$$

and

$$
\gamma_{-1}(m)=\alpha_{-1} \cos \frac{m \pi}{3}-\beta_{-1} \sin \frac{m \pi}{3} .
$$

When $m=0$,

$$
\begin{aligned}
x_{1} & =\frac{\mu}{\gamma_{0}(-1) \gamma_{-1}(0)}=\frac{\mu}{\left(\alpha_{0} \cos \frac{-\pi}{3}-\beta_{0} \sin \frac{-\pi}{3}\right)\left(\alpha_{-1}\right)} \\
& =\frac{\mu}{\frac{1}{2}\left(\alpha_{0}+\sqrt{3} \beta_{0}\right)\left(\alpha_{-1}\right)}=\frac{\mu}{x_{-1}\left(-x_{-1}+x_{-2}\right)} \\
& =\frac{x_{0} x_{-2}}{-x_{-1}+x_{-2}} .
\end{aligned}
$$


Similarly

$$
\begin{aligned}
x_{2} & =\frac{\mu}{\gamma_{0}(0) \gamma_{-1}(0)}=\frac{\mu}{\alpha_{0} \alpha_{-1}} \\
& =\frac{x_{0} x_{-1} x_{-2}}{\left(-x_{0}+x_{-1}\right)\left(-x_{-1}+x_{-2}\right)} \\
& =\frac{x_{1} x_{-1}}{-x_{0}+x_{-1}} .
\end{aligned}
$$

Suppose that the solution (3.3) is true for $m>0$.

Then

$$
\begin{aligned}
\frac{x_{2 m+1} x_{2 m-1}}{-x_{2 m}+x_{2 m-1}} & =\frac{\left(\frac{\mu}{\gamma_{0}(m-1) \gamma_{-1}(m)}\right)\left(\frac{\mu}{\gamma_{0}(m-2) \gamma_{-1}(m-1)}\right)}{-\frac{\mu}{\gamma_{0}(m-1) \gamma_{-1}(m-1)}+\frac{\mu}{\gamma_{0}(m-2) \gamma_{-1}(m-1)}} \\
& =\frac{\mu}{\gamma_{-1}(m)\left(-\gamma_{0}(m-2)+\gamma_{0}(m-1)\right)} .
\end{aligned}
$$

But we can show that

$$
\gamma_{0}(m-1)-\gamma_{0}(m-2)=\gamma_{0}(m), m=0,1, \ldots
$$

This implies that

$$
\begin{aligned}
\frac{x_{2 m+1} x_{2 m-1}}{-x_{2 m}+x_{2 m-1}} & =\frac{\mu}{\gamma_{0}(m) \gamma_{-1}(m)} \\
& =x_{2 m+2} .
\end{aligned}
$$

Similarly we can show that

$$
\frac{x_{2 m+2} x_{2 m}}{a x_{2 m+1}+b x_{2 m}}=x_{2 m+3} \text {. }
$$

This completes the proof.

It is clear for Equation (1.2) that if we start with the point $\left(x_{0}, x_{-1}, x_{-2}\right) \in \mathbb{R}^{3}$, we have the following:

If $x_{0}=0$ and $x_{-1} x_{-2} \neq 0$, then $x_{3}$ is undefined.

If $x_{-1}=0$ and $x_{0} x_{-2} \neq 0$, then $x_{5}$ is undefined.

If $x_{-2}=0$ and $x_{0} x_{-1} \neq 0$, then $x_{4}$ is undefined.

Therefore, any point $\left(x_{0}, x_{-1}, x_{-2}\right) \in \mathbb{R}^{3}$ with $x_{0} x_{-1} x_{-2}=0$ belongs to the forbidden set of Equation (1.2).

The following result provides the forbidden set of Equation (1.2).

Theorem 3.2. The forbidden set of equation (1.2) is

$$
\begin{aligned}
F= & \bigcup_{i=0}^{2}\left\{\left(u_{0}, u_{-1}, u_{-2}\right) \in \mathbb{R}^{3}: u_{-i}=0\right\} \cup\left\{\left(u_{0}, u_{-1}, u_{-2}\right) \in \mathbb{R}^{3}: u_{0}=u_{-1}\right\} \cup \\
& \left\{\left(u_{0}, u_{-1}, u_{-2}\right) \in \mathbb{R}^{3}: u_{-1}=u_{-2}\right\} .
\end{aligned}
$$

\subsection{Global Behavior of Equation (1.2)}

Theorem 3.3. Every admissible solution for Equation (1.2) is periodic with prime period six.

Proof. Suppose that $\left\{x_{n}\right\}_{n=-2}^{\infty}$ is an admissible solution for Equation (1.2).

It is clear that both the functions $\gamma_{-1}(m)$ and $\gamma_{0}(m)$ satisfy

$$
\gamma_{-1}(m+3)=-\gamma_{-1}(m) \text { and } \gamma_{0}(m+3)=-\gamma_{0}(m) \text {. }
$$

Then

$$
\begin{aligned}
x_{2(m+3)+1} & =\frac{\mu}{\gamma_{0}(m+2) \gamma_{-1}(m+3)} \\
& =\frac{\mu}{\gamma_{0}(m-1) \gamma_{-1}(m)} \\
& =x_{2 m+1}, m=-1,0, \ldots
\end{aligned}
$$

Similarly

$$
\begin{aligned}
x_{2(m+3)+2} & =\frac{\mu}{\gamma_{0}(m+3) \gamma_{-1}(m+3)} \\
& =\frac{\mu}{\gamma_{0}(m) \gamma_{-1}(m)} \\
& =x_{2 m+2}, m=-2,-1, \ldots \ldots
\end{aligned}
$$

Therefore, the solution $\left\{x_{n}\right\}_{n=-2}^{\infty}$ is periodic with prime period six. This completes the proof. 


\section{Example (3)}

Figure (3.1) shows that a solution $\left\{x_{n}\right\}_{n=-2}^{\infty}$ of equation (1.2) with $x_{-2}=-3.2, x_{-1}=2.8$ and $x_{0}=0.9$ is periodic with prime period six.

\section{Example (4)}

Figure (3.2) shows that a solution $\left\{x_{n}\right\}_{n=-2}^{\infty}$ of equation (1.2) with $x_{-2}=1.2, x_{-1}=1.7$ and $x_{0}=-0.2$ is periodic with prime period six.

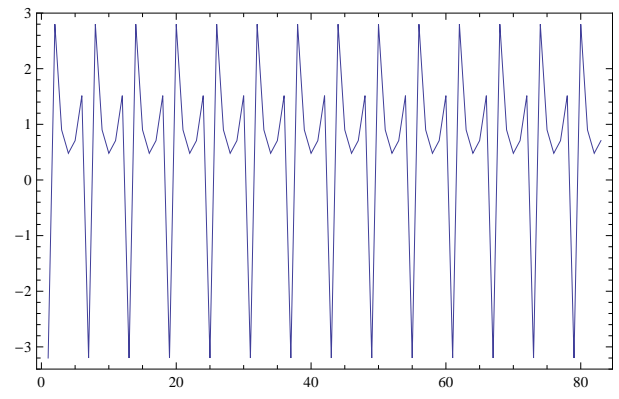

Figure 3.1: $x_{n+1}=\frac{x_{n} x_{n-2}}{-x_{n-1}+x_{n-2}}$

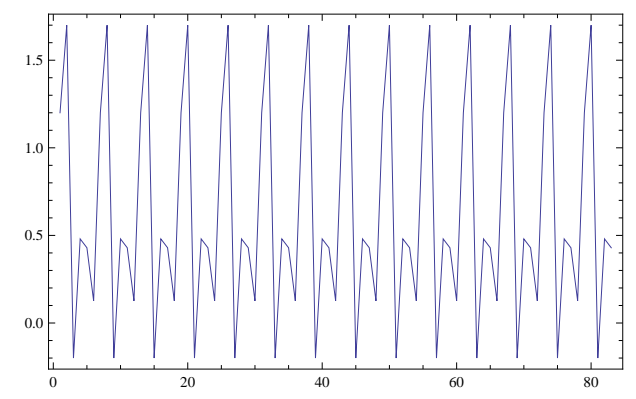

Figure 3.2: $x_{n+1}=\frac{x_{n} x_{n-2}}{-x_{n-1}+x_{n-2}}$

\section{References}

[1] R. Abo-Zeid, Behavior of solutions of a second order rational difference equation, Math. Morav., 23 (1) (2019), $11-25$.

[2] R. Abo-Zeid, Global behavior of two third order rational difference equations with quadratic terms, Math. Slovaca, 69 (1) (2019), 147-158 .

[3] R. Abo-Zeid, Global Behavior of a fourth order difference equation with quadratic term, Bol. Soc. Mat. Mexicana, 25 (1) (2019), 187-194 .

[4] R. Abo-Zeid, Behavior of solutions of a higher order difference equation, Alabama J. Math., 42 (2018), 1-10.

[5] R. Abo-Zeid, On the solutions of a higher order difference equation, Georgian Math. J., doi:10.1515/gmj-2018-0008.

[6] R. Abo-Zeid, On a third order difference equation, Acta Univ. Apulensis, 55 (2018), 89-103 .

[7] R. Abo-Zeid Forbidden sets and stability in some rational difference equations, J. Difference Equ. Appl., 24 (2) (2018), $220-239$.

[8] R. Abo-Zeid, On the solutions of a second order difference equation, Math. Morav., 21 (2) (2017), 61-75.

[9] R. Abo-Zeid, Global behavior of a higher order rational difference equation, Filomat 30 (12) (2016), 3265-3276

[10] R. Abo-Zeid, Global behavior of a third order rational difference equation, Math. Bohem., 139 (1) (2014), $25-37$.

[11] R. Abo-Zeid, On the solutions of two third order recursive sequences, Armenian J. Math., 6 (2) (2014), 64-66.

[12] R. Abo-Zeid, Global behavior of a fourth order difference equation, Acta Commentaiones Univ. Tartuensis Math., 18 (2) (2014), $211-220$.

[13] A.M. Amleh, E. Camouzis and G. Ladas, On the dynamics of a rational difference equation, Part 2, Int. J. Difference Equ., 3 (2) (2008), $195-225$.

[14] A.M. Amleh, E. Camouzis and G. Ladas, On the dynamics of a rational difference equation, Part 1, Int. J. Difference Equ., 3 (1) (2008), 1-35 .

[15] I. Bajo, Forbidden sets of planar rational systems of difference equations with common denominator, Appl. Anal. Discrete Math., 8 (2014), 16-32

[16] F. Balibrea and A. Cascales, On forbidden sets, J. Difference Equ. Appl. 21 (10) (2015), 974-996.

[17] E. Camouzis and G. Ladas, Dynamics of Third Order Rational Difference Equations: With Open Problems and Conjectures, Chapman \& Hall/CRC, Boca Raton, 2008

[18] H. El-Metwally and E. M. Elsayed, Qualitative study of solutions of some difference equations, Abstr. Appl. Anal., 2012 (2012), Article ID 248291 , 16 pages, doi: 10.1155/2012/248291.

[19] E.M. Elsayed, Solution and attractivity for a rational recursive sequence, Discrete Dyn. Nat. Soc., 2011 (2011), Article ID 982309 , 18 pages, doi: $10.1155 / 2011 / 982309$.

[20] M. Gümüş, The global asymptotic stability of a system of difference equations, J. Difference Equ. Appl., 24 (6) (2018) , 976-991 .

[21] M. Gümüş and Ö. Öcalan, The qualitative analysis of a rational system of diffrence equations, J. Fract. Calc. Appl., 9 (2) (2018), 113-126 .

[22] Inci Okumuss and Yüksel Soykan, A review on dynamical nature of systems of nonlinear difference equations, J. Inform. Math. Sci., 11 (2) (2019), $235-251$.

[23] R. Khalaf-Allah, Asymptotic behavior and periodic nature of two difference equations, Ukrainian Math. J., 61 (6) (2009), 988-993 .

[24] V. L. Kocic, G. Ladas, Global Behavior of Nonlinear Difference Equations of Higher Order with Applications, Kluwer Academic, Dordrecht, 1993 .

[25] M. R. S. Kulenović, and M. Mehuljić, Global behavior of some rational second order difference equations, Int. J. Difference Equ., 7 (2) (2012), $153-162$.

[26] M.R.S. Kulenović and G. Ladas, Dynamics of Second Order Rational Difference Equations: With Open Problems and Conjectures, Chapman and Hall/HRC, Boca Raton, 2002.

[27] H. Sedaghat, On third order rational equations with quadratic terms, J. Difference Equ. Appl., 14 (8) (2008) , $889-897$.

[28] I. Szalkai, Avoiding forbidden Sequences by finding suitable initial values, Int. J. Difference Equ., 3 (2) (2008), $305-315$. 